\title{
Antenna-enhanced high harmonic generation in a wide-bandgap semiconductor $\mathrm{ZnO}$
}

\author{
Kotaro Imasaka ${ }^{1}$, Tomohiro Kaji ${ }^{1}$, Tsutomu Shimura $^{1}$, and Satoshi Ashihara ${ }^{1,{ }^{*}}$ \\ ${ }^{1}$ Institute of Industrial Science, The University of Tokyo, 4-6-1, Komaba, Meguro-ku, Tokyo, 153- \\ 8505 , Japan
}

\begin{abstract}
We demonstrate high harmonic generation (HHG) into deep$\mathrm{UV}$ range in a $\mathrm{ZnO}$ single crystal with resonant nanoantennas. Nonperturbative HHG is successfully induced by optical excitation of as low as $20 \mathrm{GW} / \mathrm{cm}^{2}$. The spectral selection rule is found to reflect crystal symmetry, suggesting the possibility of nano-scaled EUV sources and band-structure reconstruction.
\end{abstract}

\section{Introduction}

High harmonic generation in solids under the non-perturbative regime has been observed with few-cycle mid-infrared (MIR) optical electric-field exceeding $1 \mathrm{~V} / \mathrm{nm}$ [1]. The mechanism is described as inter-band (Zener) tunnelling, acceleration of crystal electrons/holes, and the following high harmonic $(\mathrm{HH})$ radiation via intra-band current and/or inter-band polarization. Because solids have high atomic density, crystal symmetry, and characteristic band structures, solid $\mathrm{HHG}$ is expected to have great potential for efficient EUV attosecond sources, all-optical band-structure reconstruction, probing electron dynamics, and to develop condensed-phase attosecond science.

A smart way to obtain intense optical field for driving the strong-field phenomena is to confine electromagnetic fields by using nanostructures [2]. Recently, Au-coated sapphire nano-cone [3], $\mathrm{ZnO}$ nano-cone [4], and silicon $\left(E_{\mathrm{g}}=1.2 \mathrm{eV}\right)$ with metallic nanoantennas [5] have been utilized to generate odd-order harmonic radiations. Here, we demonstrate antenna-enhanced HHG into deep-UV (DUV) range (9th, $222 \mathrm{~nm})$ from $\mathrm{ZnO}\left(E_{\mathrm{g}}=3.4 \mathrm{eV}\right)$, driven by MIR femtosecond pulses. Non-perturbative HHG is successfully induced by optical excitation of as low as $20 \mathrm{GW} / \mathrm{cm}^{2}$. Even-order as well as odd-order harmonics are observed, when the nanoantennas are oriented along the symmetry-broken crystal axis. Our results indicate that the spectral selection rule of high harmonics precisely reflects the crystal symmetry of the substrate material.

\section{Devices and methods}

A $2 \mathrm{D}$ array of a rod-shaped Au nanoantenna is fabricated on a 0.5 -mm-thick $a$-cut $\mathrm{ZnO}$ crystal by electron-beam lithography (SEM image in Fig. 1(a)). The size and period of the

\footnotetext{
*Corresponding author: ashihara@iis.u-tokyo.ac.jp
} 
antennas are designed for the long axis to be resonant at the wavelength of $2 \mu \mathrm{m}$. An FDTD simulation predicts the near-field distribution shown in Fig. 1(b), with an intensity enhancement factor of $\sim 80$ at the hotspots. The extinction (experimental) and enhancement factor (numerical) spectrum of the nanoantenna array well cover the pulse spectrum so that the incident waveform is maintained (Fig. 1(c)) [6]. To investigate the dependence of the HHG properties on crystal symmetry, each nanoantenna array is aligned parallel to either $m$-axis (sample I) or $c$-axis (sample II).

The excitation light-source is a MIR pulse $(2 \mu \mathrm{m}, 100 \mathrm{fs})$, generated from a femtosecond OPA. The samples are exposed by the linearly-polarized MIR pulse parallel to the long axis of the antennas at normal incidence. The HHs parallel to incident polarization are recorded with a grating spectrometer.

(a)

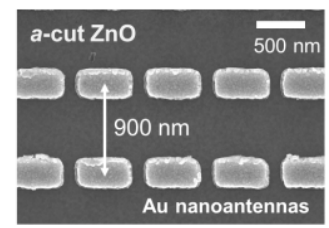

(b)

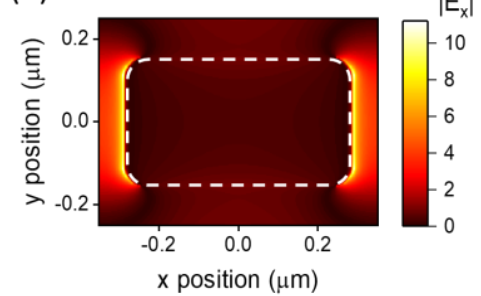

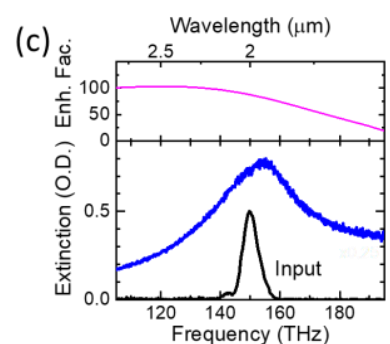

Fig. 1. (a) A SEM image of an antenna array fabricated on $\mathrm{ZnO}$. (b) A simulated near-field distribution of $|\mathrm{Ex}|$ inside the $\mathrm{ZnO}$ substrate (2-nm beneath the air- $\mathrm{ZnO}$ interface). The dashed line shows the antenna area. (c) The measured extinction spectrum (blue) is shown with the calculated intensity enhancement factor spectrum (pink). A black line represents the input pulse spectrum.

\section{Results and discussions}

Figures $2(\mathrm{a}, \mathrm{b})$ show the $\mathrm{HH}$ spectrum of a $\mathrm{ZnO}$ crystal with/without nanoantennas, for the sample I ( $E_{\text {in }} / / m$-axis $)$ and for the sample II $\left(E_{\text {in }} / / c\right.$-axis $)$ respectively. Here the excitation peak intensity is $0.10 \mathrm{TW} / \mathrm{cm}^{2}$. In contrast to that HHGs from the bulk $\mathrm{ZnO}$ samples without nanoantennas extend up to 7th order, HHGs from the samples I and II with nanoantennas extend up to 9th order $(222 \mathrm{~nm})$ including the bandgap fluorescence (FL). We can see that each of the 5, 6, 7, 8, and 9th-order harmonics is well enhanced (especially more than two-order enhancement for 9th order harmonic). Whereas only odd-order harmonics are observed for the sample I, both odd- and even-order harmonics are observed for the sample II. In this way, we can conclude that the spectral selection rule of high harmonics is intrinsically governed by the crystal symmetry of $\mathrm{ZnO}$.

Figure 2(c) displays the drive-laser intensity dependence of the 7 th harmonic spectral energy for the sample II with/without antennas with a line expressing the multi-photon dependence. One can see that the onset of HHG shifts to lower intensity due to the antennas, and that enhancement of HHG is more remarkable at weaker excitation. Bulk HHG follows the multi-photon law at lower intensity but deviates from it at higher intensity. Such kink in the slope indicates the transition from the perturbative to the non-perturbative regime. On the contrary, the slope for the antenna-enhanced case is smaller than 7 even at $\sim 0.02$ $\mathrm{TW} / \mathrm{cm}^{2}$. This fact indicates that the plasmonic near-field is strong enough to induce the non-perturbative HHG at such low illumination.

The clear increase in $\mathrm{HH}$ intensity due to the nano-scaled plasmonic near-fields indicates substantial local enhancements of HHG. By considering the hotspot volume, the local enhancement factor is estimated to be $10^{3}-10^{5}$ for 7 th- and 9 th-order harmonics. 
(a)

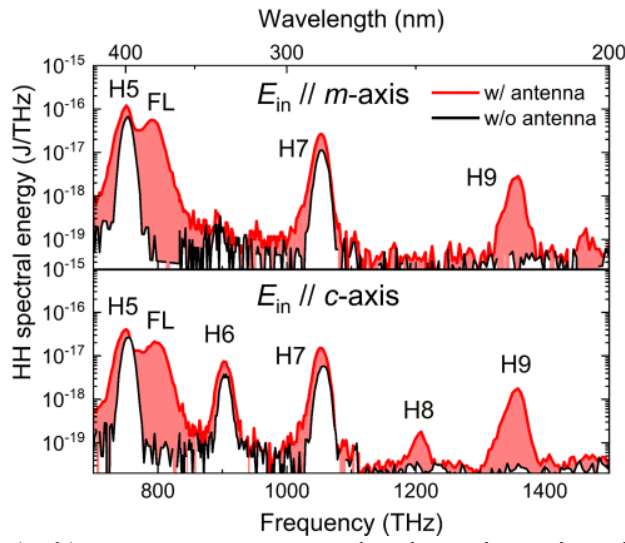

(c)

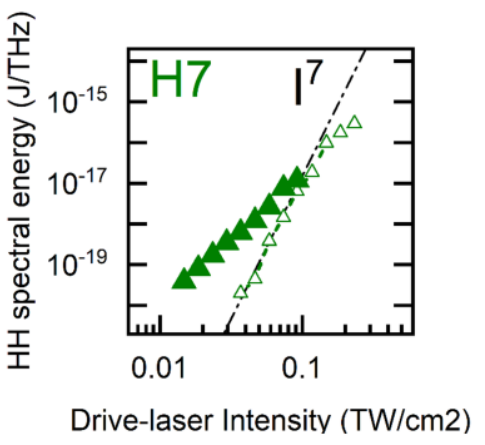

Fig. 2. $(\mathrm{a}, \mathrm{b}) \mathrm{HH}$ spectra measured at input intensity of $0.10 \mathrm{TW} / \mathrm{cm}^{2}$ for (a) the sample $\mathrm{I}\left(E_{\text {in }} / / \mathrm{m}\right.$ axis) and (b) the sample II ( $E_{\text {in }} / / c$-axis). The red (black) lines indicate the HH spectra generated from $\mathrm{ZnO}$ with (without) antennas. (c) Drive-laser intensity dependence of the 7th harmonic spectral energy, measured for the sample II ( $E_{\text {in }} / / c$-axis) with (filled markers) and without (open markers) antennas. The dot-dashed line expresses the multi-photon law.

\section{Conclusions}

We demonstrate antenna-enhanced HHG into DUV range in a $\mathrm{ZnO}$ crystal using MIR femtosecond pulses with an intensity of as low as $20 \mathrm{GW} / \mathrm{cm}^{2}$. In the presence of the antennas, orders-of-magnitude enhanced conversion efficiency at the hot-spots brings about ten-fold enhancement in the total harmonic yield. We successfully generate even-order harmonics as well as odd-order harmonics by resonantly-enhanced plasmonic near-fields, by orienting resonant nanoantennas along the symmetry-broken crystal axis. The results indicate that the spectral selection rule precisely reflects the crystal symmetry and that $\mathrm{HH}$ radiations are dominantly generated from a $\mathrm{ZnO}$ substrate, not from metallic nanoantennas. Nanoantennas will be useful for the realization of HHG driven by compact light-sources, precise control of $\mathrm{HH}$ properties, and various strong-field phenomena in solids.

This paper was financially supported by Japan Society for the Promotion of Science (JSPS) (MEXT KAKENHI 16K13694, 18K19030, Bilateral Joint Research Projects 1007490).

\section{References}

1. S. Ghimire, A. D. DiChiara, E. Sistrunk, P. Agostini, L. F. DiMauro, D. A. Reis, Nat. Phys. 7, 138 (2011).

2. F. Kusa, K. E. Echternkamp, G. Herink, C. Ropers, S. Ashihara, AIP Adv. 5, 077138 (2015).

3. S. Han, H. Kim, Y. W. Kim, Y. J. Kim, S. Kim, I. Y. Park, and S. W. Kim, Nat. Commun. 7, 13105 (2016).

4. M. Sivis, M. Taucer, G. Vampa, K. Johnston, A. Staudte, A. Y. Naumov, D. M. Villeneuve, C. Ropers, P. B. Corkum, Science 357, 303-306 (2017).

5. G. Vampa, B. G. Ghamsari, S. S. Mousavi, T. J. Hammond, A. Olivieri, E. Lisicka-Skrek, A. Yu Naumov, D. M. Villeneuve, A. Staudte, P. Berini, P. B. Corkum, Nat. Phys. 13, 659 (2017).

6. F. Kusa, S. Ashihara, J. Appl. Phys. 116, 153103 (2014). 